\title{
Analgesic effect of light-emitting diode phototherapy on incised wound is via inhibiting IL-6, COX-2 and PGE-2 but not IL1- $\beta$ and TNF- $\alpha$
}

\author{
Ping-Heng Tan, Chien-Cheng Liu \\ Department of Anesthesiology, E-DA Hospital/I-Shou University, Kaohsiung, Taiwan
}

\section{Introduction:}

Light-emitting diode (LED) phototherapy has been reported to relieve pain and enhance tissue repair through several mechanisms. The treatment of postsurgical pain remains a challenge for physicians. The analgesic effects of LEDs on incised skin wounds have never been examined. In this study, we examined the analgesic effects of LED therapy on skin incision pain and measured changes in cyclo-oxygenase 2 (COX-2), prostaglandin E2 (PGE2) and pro-inflammatory cytokines (interleukin (IL)-6, IL1- $\beta$, and tumor necrosis factor $\alpha$ (TNF- $\alpha$ )).

\section{Material and Methods:}

The rats were randomly assigned to the following groups. Rats received LED therapy 6 days before incision (LI group) or 6 days after incision (IL group) or from 3 days before incision to 3 days after incision (LIL group) and skin incision only (I group).

LED (940 $10 \mathrm{~nm}$ ) CW on the lesion area of the right limb of the animal. The application time is 30 minutes, with a dose of energy measured on the source of $7.5 \mathrm{~J} / \mathrm{cm} 2$ and applied on the injured area.

Pain behavior was tested by measuring mechanical withdrawal threshold for mechanical allodynia and thermal withdrawal latency for thermal hyperalgesia. After behavioral testing, the skin tissues were collected for COX-2, PGE2, IL-6, IL1- $\beta$ and TNF- $\alpha$ protein analyses ( $\mathrm{n}=6$ each group).

\section{Results:}

The thermal withdrawal latency was significantly decreased in the incision-only group $(p=0.013)$ but not in the three LED treatment groups. Significantly decreased mechanical withdrawal thresholds were noted after skin incision in all LED treatment groups $(p<0.05)$. The expression of IL-6, COX-2 and PGE2 were significantly decreased in the three LED-treated groups compared to the I group $(p<0.05)$. IL1- $\beta$ and TNF- $\alpha$ were significantly decreased in the LI group $(<0.05)$ but not in the other two LED-treated groups.

Conclusions: LED therapy relieved thermal hyperalgesia but not mechanical allodynia in incised wounds, and this analgesic effect was possibly produced by the inhibition of IL-6, COX-2 and PGE2 expression.

\section{Discussion:}

1. Light irradiation can disrupt the ROS directly and subsequently inhibits expression of CPLA2, SPLA2, and COX, resulting in inhibition of PGE-2 release.

2. Some portion of TNF-a and IL-1ß were secreted by Schwann cells that were not inhibited by LED irradiation.

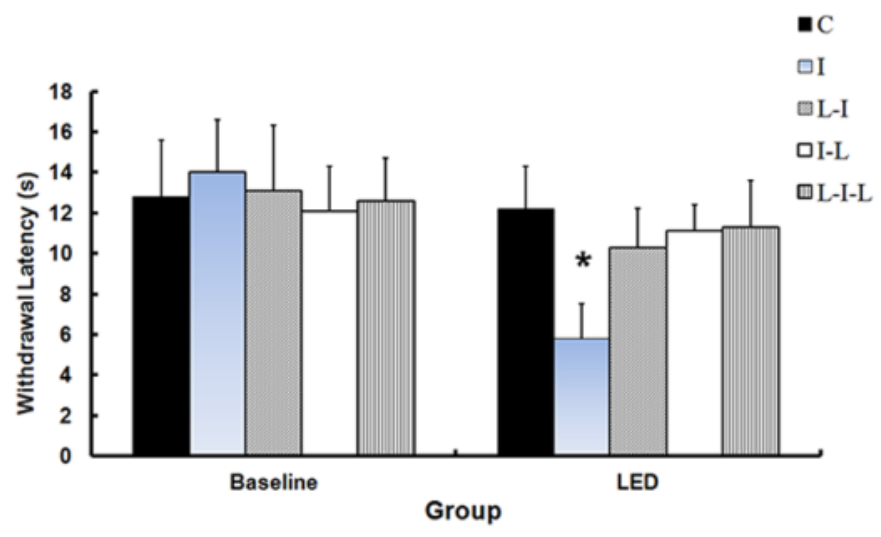

Fig.1 Mean withdrawal latency in the five groups ( $n=6$ each group) after LED irradiation performed before or after skin incision.

a

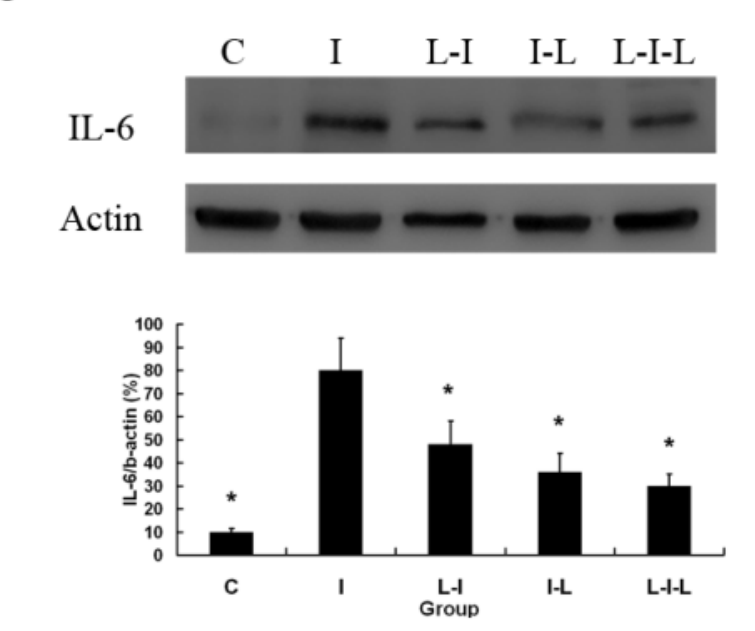

b
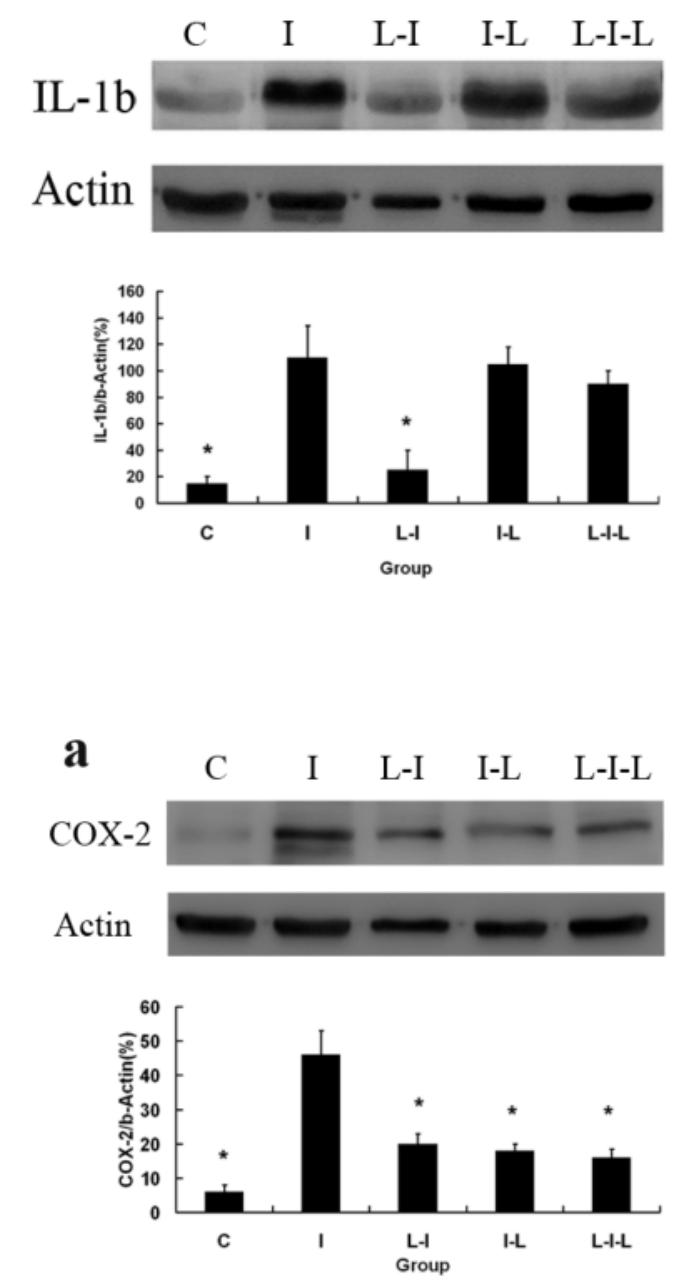

b

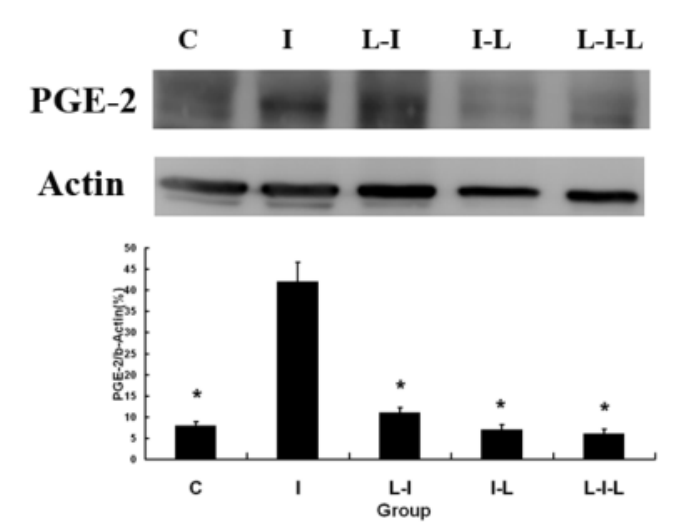

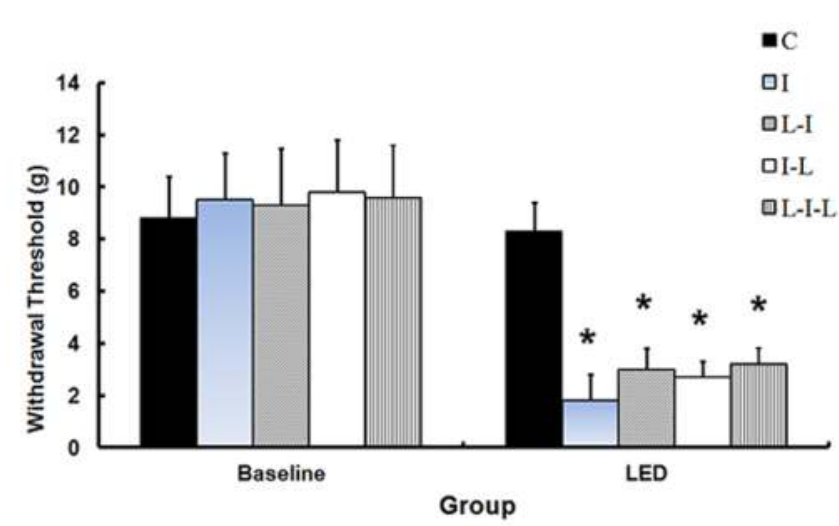

Fig.2 Mean withdrawal threshold in the six groups ( $n=6$ each group) after LED irradiation performed before or after skin incision.

C

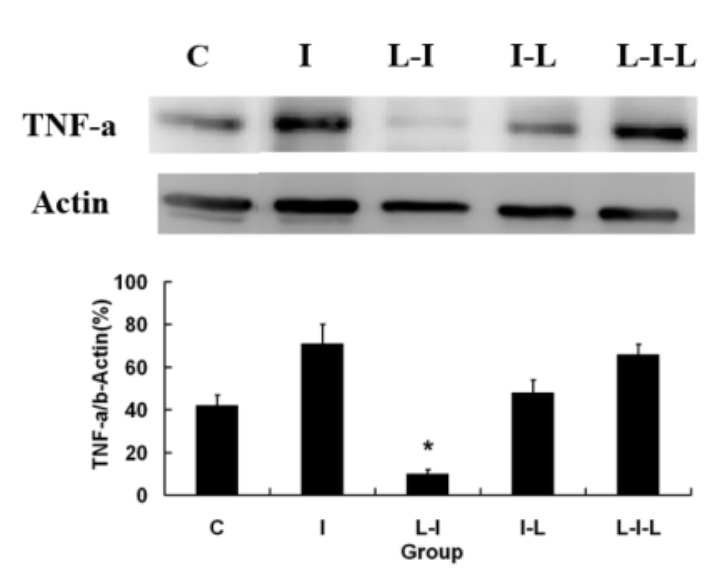

Fig.3 Representative Western blots of interleukin-6 (IL-6), IL1- $\beta$ and tumor necrosis factor (TNF)- $\alpha$ after LED irradiation.

Fig.4 Representative Western blots of cyclooxygenase-2 (COX-2) and prostaglandin E2 (PGE-2) expression after LED irradiation.

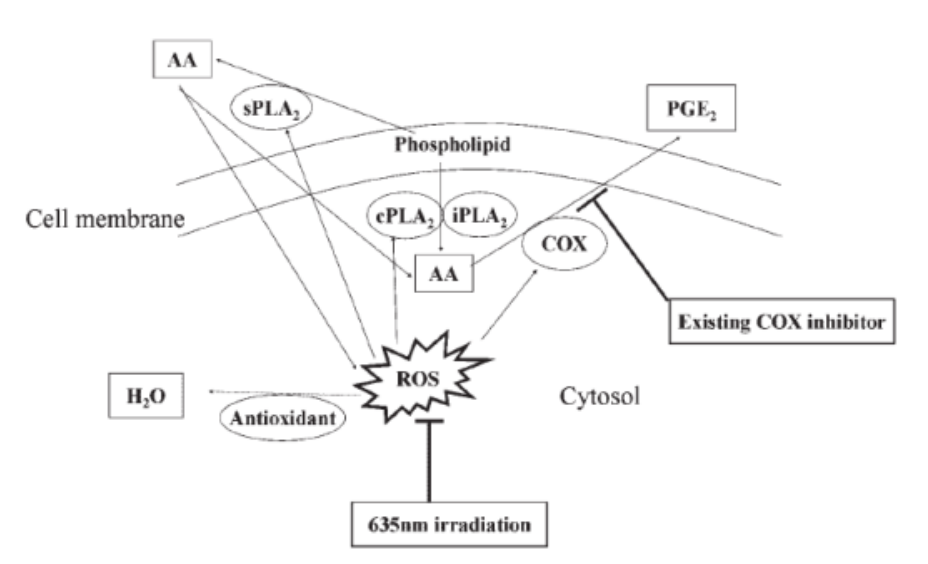

